\title{
Targeting the reversible drug-tolerant state: aurora kinase $A$, is that the final answer?
}

\author{
Kenichi Suda \\ Division of Thoracic Surgery, Department of Surgery, Kindai University Faculty of Medicine, Osaka-Sayama, Japan \\ Correspondence to: Kenichi Suda, MD, PhD. Division of Thoracic Surgery, Department of Surgery, Kindai University Faculty of Medicine, 377-2 \\ Ohno-higashi, Osaka-Sayama 589-8511, Japan. Email: ascaris@surg2.med.kyushu-u.ac.jp. \\ Comment on: Shah KN, Bhatt R, Rotow J, et al. Aurora kinase A drives the evolution of resistance to third-generation EGFR inhibitors in lung cancer. \\ Nat Med 2019;25:111-8.
}

Submitted May 12, 2019. Accepted for publication May 19, 2019.

doi: $10.21037 /$ tcr.2019.05.21

View this article at: http://dx.doi.org/10.21037/tcr.2019.05.21

Clinical application of the first-generation (1G) epidermal growth factor receptor (EGFR) tyrosine kinase inhibitors (TKIs) gefitinib and erlotinib launched the era of molecular targeted therapies for lung adenocarcinoma. At the same time, acquired resistance to these agents has been problematic given that $1 \mathrm{G}$ EGFR-TKIs are associated with a median progression-free survival of 9-12 months (1).

The mechanistic origins of acquired resistance to EGFRTKIs are considered to be multifocal as summarized in our previous review (2). In that review, we classified the origins into three categories: (I) pre-existing minor subpopulation with a genetic resistance mechanism; (II) reversible (nongenetic) drug-tolerant state; (III) roles of the tumor microenvironment such as cancer-associated fibroblasts/ dying cancer cells and poor vascularization/hypoxic condition.

In the era of $1 \mathrm{G}$ or $2 \mathrm{G}$ EGFR-TKIs, i.e., before the approval of front-line osimertinib in 2018, the most problematic acquired resistance mechanism was the EGFR T790M secondary mutation (3). One origin of this mutation is that many EGFR-mutated lung cancers possess a pre-existing minor subpopulation carrying the T790M mutation. For example, using an ultrasensitive detection method, up to $79.9 \%$ of $E G F R$-mutated lung cancers were found to carry a pre-treatment T790M mutation (4). The allele frequency of the EGFR T790M mutation ranged between 0.001 and $0.1 \%$ in most cases (95\%) (4). Why can cancer cells "prepare" this resistance mutation before the initiation of $1 \mathrm{G}$ or $2 \mathrm{G}$ EGFR-TKI therapy? Although the evidence is not solid, Fujii and colleagues suggested that hypermethylation of the CpG dinucleotide in EGFR codon 790 leads to the C-to- $\mathrm{T}$ transition mutation, causing the T790M mutation (5).

However, the 3G EGFR-TKI osimertinib has changed this dynamic because it is also active against lung cancers featuring the EGFR T790M mutation (6). This means that EGFR-mutated lung cancer cells cannot evade therapy based on the presence of a pre-existing minor subpopulation with T790M mutation. Therefore, it can be hypothesized that drug resistance originating from (II) a reversible (nongenetic) drug-tolerant state or related to (III) the tumor microenvironment will be more important in the era of front-line osimertinib.

The first attempt to combat the reversible drug-tolerant state was reported in 2010 by Sharma and colleagues (7). In this study, which mainly used the PC9 cell line, the authors observed that a small fraction of viable, largely quiescent cells [named drug-tolerant persisters (DTPs)] remained detectable after 9 days in the presence of an EGFR-TKI (gefitinib or erlotinib). DTPs had similar characteristics as cancer stem cells (higher CD133 and CD24 levels than the parental PC9 cells). However, the DTPs eventually 
resumed normal proliferation in the presence of the drug (upregulated cancer stem cell markers were decreased), and the authors referred to these cells as drug-tolerant expanded persisters (DTEPs). Through analyses of DTPs and DTEPs, the authors identified that drug-tolerant cells required the histone demethylase KDM5A/RBP2/Jarid1A, and they were selectively ablated by HDAC inhibitors. In addition, DTPs were also sensitive to insulin-like growth factor 1 receptor (IGF-1R) inhibition, and combination therapy with an IGF-1R inhibitor (AEW541) and an EGFR-TKI eliminated these drug-tolerant cells. Following this discovery, many research groups identified a long list of candidate molecules related to the drug-tolerant state upon EGFR-TKI therapy in lung cancer cells with EGFR mutation; e.g., NF- $\mathrm{B}, \beta$-catenin, STAT3, Ets1 , endoplasmic reticulum (ER) stress signaling, glucose metabolism, MCL-1, and AXL (8-20).

The next breakthrough in this field was made by Hata and colleagues, who demonstrated that cells with acquired resistance associated with genetic changes such as the T790M mutation evolve from drug-tolerant cells (lacking the T790M mutation), highlighting the importance of targeting drug-tolerant cells (21). By what mechanism can drug-tolerant cells acquire a genetic change (T790M mutation) that causes irreversible drug resistance? One possible mechanism was reported by El Kadi and colleagues (22). EGFR-TKI therapy led to activation of the NF- $\mathrm{KB}$ pathway, thereby inducing the expression of activation-induced cytidine deaminase, which generated the T790M mutation by inducing the deamination of 5-methylcytosine to thymine at position c.2369.

Following these studies, in 2019, Shah and colleagues reported that non-genetic resistance through the activation of aurora kinase A (AURKA) by its coactivator TPX2 emerges in response to osimertinib monotherapy by preventing drug-induced apoptosis (23). Via compound screening (a 94-compound cancer-focused library), they achieved synergistic inhibition of AURKA inhibitors with osimertinib or another 3G EGFR-TKI, namely rociletinib, using four EGFR-mutated lung cancer cell lines (PC9, HCC827, HCC4006, and NCI-H1975) after chronic EGFR-TKI exposure with stepwise dose escalation over a 9-day period followed by maintenance treatment at a dose of $1 \mu \mathrm{M}$ for 6 weeks. In addition to experimental models, the authors observed that TPX2 expression was significantly increased [Immunohistochemistry (IHC) score $>2$ ) in six of nine paired tumor tissue samples obtained after EGFRTKI treatment failure compared with the findings in pretreatment samples. Interestingly, the importance of AURK inhibition in enhancing apoptosis upon EGFR-TKI therapy in EGFR-mutated lung cancer cells was also described at the recent 2019 AACR annual meeting by another research group (24). Are these reports just adding one molecule at the end of the aforementioned long list of candidate molecules related to the drug-tolerant state in lung cancer cells with EGFR mutation, or is AURK the final target for combination therapy featuring osimertinib to suppress drug-tolerant cells? At the end of this commentary, I would like to raise three points that would support the importance of AURKA over other candidate molecules.

First, it is easy to understand why some cancer cells have high AURKA activation at the time of EGFR-TKI therapy. It is well known that in G2/M phase, cells have high levels of phosphorylated AURKA, and in fact, Shah and colleagues also reported that parental PC9 cells synchronized into G2/ $M$ phase using serum starvation or thymidine block were less sensitive to EGFR-TKIs than cells in G1/S phase or asynchronous cells (23). The second advantage of AURKA inhibition in combination with osimertinib is that many AURK inhibitors are currently in clinical development (25). Last, as illustrated in Figure 1, AURKA is related to many molecules that have been reported as causes of drug-tolerant states. Therefore, it is possible that AURKA inhibition can be a "master key" to repress important signaling pathways for drug-tolerant cells at an early phase of EGFR-TKI treatment. 


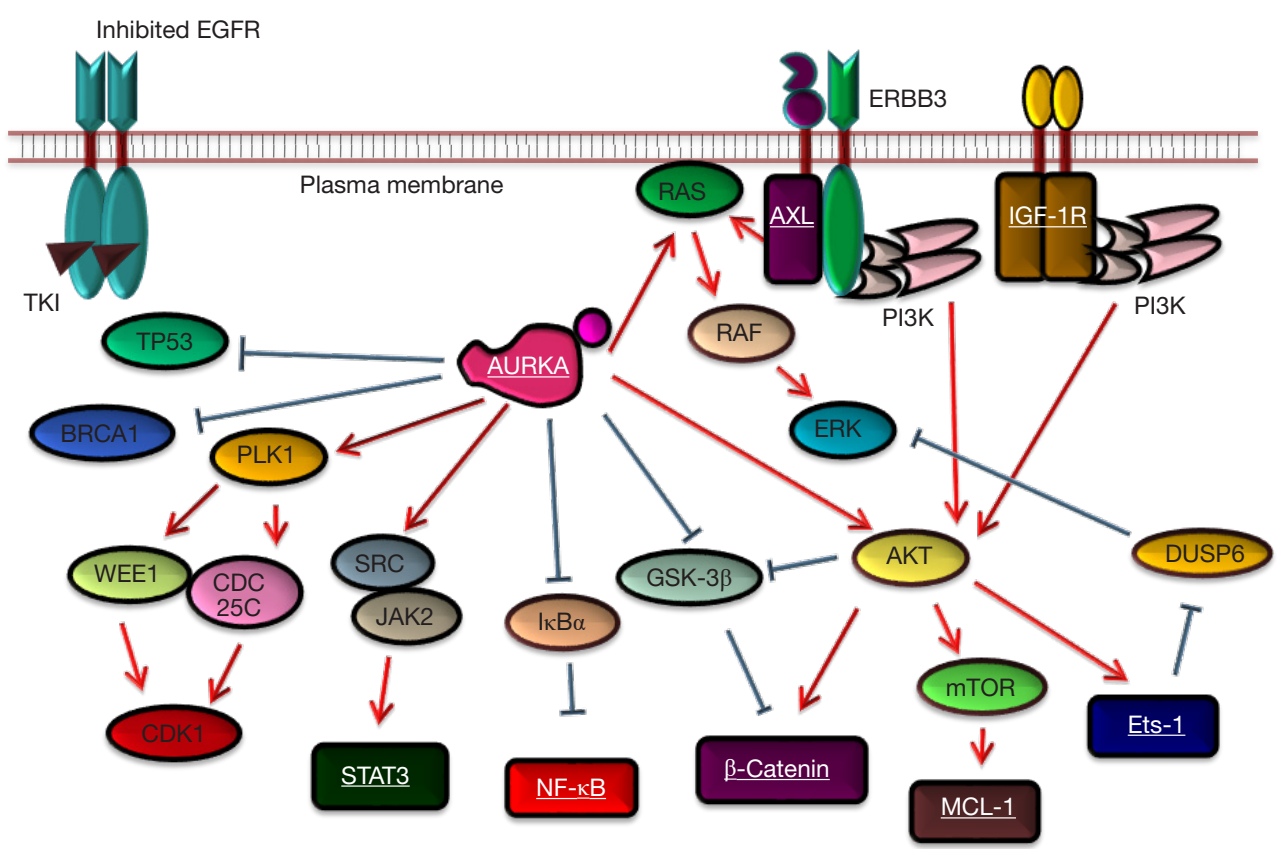

Figure 1 Scheme of the AURKA pathway and molecules that are reported to cause the drug-tolerant state [AXL (20), IGF-1R (7,21), NF- $\mathrm{B}(8,9,13,14,17), \beta$-catenin (10), STAT3 (11,12), Ets-1 (15), and MCL-1 (18), which are highlighted in white bold letters] in lung cancer cells carrying EGFR mutations upon EGFR-TKI treatment. It is interesting that the compound screening by Shah and colleagues also identified that compounds with the 3rd to 7th highest synergy scores were inhibitors of PI3K, AKT, mTOR, or Src (although they are not highlighted, they may have some roles in the drug-tolerant state; as a side note, the compounds with the highest two synergy scores were AURK inhibitors) (23). Some other candidate factors that might be related to drug tolerance; e.g., altered chromatin state (7), ER stress signaling (19), or glucose metabolism (16), are not illustrated in this figure; however, it is noteworthy that AURKA inhibition will lead to the inhibition of multiple potential causes of drug tolerance. The figure was generated using a combination of results from multiple published works related to the AURKA pathway (26-29) or drug tolerance states (15,18,20,23); therefore, it is possible that it may overestimate the roles of AURKA. AURKA, aurora kinase A; EGFR, epidermal growth factor receptor; TKIs: tyrosine kinase inhibitors; ER, endoplasmic reticulum.

\section{Acknowledgments}

I thank Joe Barber Jr., PhD, from Edanz Group (www. edanzediting.com/ac) for editing a draft of this manuscript. Funding: This work was supported by a Grant-in-Aid for Scientific Research from the Japan Society for the Promotion of Science (18K07336 to K Suda).

\section{Footnote}

Provenance and Peer Review: This article is commissioned and reviewed by the Section Editor Wei Xu (Division of Respiratory Disease, Department of Geriatrics, the First Affiliated Hospital of Nanjing Medical University, Nanjing, China).
Conflicts of Interest: The author has completed the ICMJE uniform disclosure form (available at http://dx.doi. org/10.21037/tcr.2019.05.21). The author reports personal fees from AstraZeneca, grants from Rain Therapeutics, grants and personal fees from Boehringer Ingelheim, outside the submitted work.

Ethical Statement: The author is accountable for all aspects of the work in ensuring that questions related to the accuracy or integrity of any part of the work are appropriately investigated and resolved.

Open Access Statement: This is an Open Access article distributed in accordance with the Creative Commons 
Attribution-NonCommercial-NoDerivs 4.0 International License (CC BY-NC-ND 4.0), which permits the noncommercial replication and distribution of the article with the strict proviso that no changes or edits are made and the original work is properly cited (including links to both the formal publication through the relevant DOI and the license). See: https://creativecommons.org/licenses/by-nc-nd/4.0/.

\section{References}

1. Hirsch FR, Suda K, Wiens J, et al. New and emerging targeted treatments in advanced non-small-cell lung cancer. Lancet 2016;388:1012-24.

2. Suda K, Bunn PA, Jr., Rivard CJ, et al. Primary DoubleStrike Therapy for Cancers to Overcome EGFR Kinase Inhibitor Resistance: Proposal from the Bench. J Thorac Oncol 2017;12:27-35.

3. Suda K, Mizuuchi H, Maehara Y, et al. Acquired resistance mechanisms to tyrosine kinase inhibitors in lung cancer with activating epidermal growth factor receptor mutationdiversity, ductility, and destiny. Cancer Metastasis Rev 2012;31:807-14.

4. Watanabe M, Kawaguchi T, Isa S, et al. Ultra-Sensitive Detection of the Pretreatment EGFR T790M Mutation in Non-Small Cell Lung Cancer Patients with an EGFRActivating Mutation Using Droplet Digital PCR. Clin Cancer Res 2015;21:3552-60.

5. Fujii A, Harada T, Iwama E, et al. Hypermethylation of the $\mathrm{CpG}$ dinucleotide in epidermal growth factor receptor codon 790: implications for a mutational hotspot leading to the T790M mutation in non-small-cell lung cancer. Cancer Genet 2015;208:271-8.

6. Mok TS, Wu YL, Ahn MJ, et al. Osimertinib or PlatinumPemetrexed in EGFR T790M-Positive Lung Cancer. N Engl J Med 2017;376:629-40.

7. Sharma SV, Lee DY, Li B, et al. A chromatin-mediated reversible drug-tolerant state in cancer cell subpopulations. Cell 2010;141:69-80.

8. Bivona TG, Hieronymus H, Parker J, et al. FAS and NFkappaB signalling modulate dependence of lung cancers on mutant EGFR. Nature 2011;471:523-6.

9. Sakuma Y, Yamazaki Y, Nakamura Y, et al. NF-kappaB signaling is activated and confers resistance to apoptosis in three-dimensionally cultured EGFR-mutant lung adenocarcinoma cells. Biochem Biophys Res Commun 2012;423:667-71.
10. Casás-Selves M, Kim J, Zhang Z, et al. Tankyrase and the canonical Wnt pathway protect lung cancer cells from EGFR inhibition. Cancer Res 2012;72:4154-64.

11. Kim SM, Kwon OJ, Hong YK, et al. Activation of IL-6R/ JAK1/STAT3 signaling induces De Novo resistance to irreversible EGFR inhibitors in non-small cell lung cancer with T790M resistance mutation. Mol Cancer Ther 2012;11:2254-64.

12. Lee HJ, Zhuang G, Cao Y, et al. Drug resistance via feedback activation of Stat 3 in oncogene-addicted cancer cells. Cancer Cell 2014;26:207-21.

13. Blakely CM, Pazarentzos E, Olivas V, et al. NF-kappaBactivating complex engaged in response to EGFR oncogene inhibition drives tumor cell survival and residual disease in lung cancer. Cell Rep 2015;11:98-110.

14. Lantermann AB, Chen D, McCutcheon K, et al. Inhibition of Casein Kinase 1 Alpha Prevents Acquired Drug Resistance to Erlotinib in EGFR-Mutant Non-Small Cell Lung Cancer. Cancer Res 2015;75:4937-48.

15. Phuchareon J, McCormick F, Eisele DW, et al. EGFR inhibition evokes innate drug resistance in lung cancer cells by preventing Akt activity and thus inactivating Ets-1 function. Proc Natl Acad Sci U S A 2015;112:E3855-63.

16. Kunimasa K, Nagano T, Shimono Y, et al. Glucose metabolism-targeted therapy and withaferin A are effective for epidermal growth factor receptor tyrosine kinase inhibitor-induced drug-tolerant persisters. Cancer Sci 2017;108:1368-77.

17. Fukuoka M, Yoshioka K, Hohjoh H. NFkappaB activation is an early event of changes in gene regulation for acquiring drug resistance in human adenocarcinoma PC-9 cells. PLoS One 2018;13:e0201796.

18. Song KA, Hosono Y, Turner C, et al. Increased Synthesis of MCL-1 Protein Underlies Initial Survival of EGFR-Mutant Lung Cancer to EGFR Inhibitors and Provides a Novel Drug Target. Clin Cancer Res 2018;24:5658-72.

19. Terai H, Kitajima S, Potter DS, et al. ER Stress Signaling Promotes the Survival of Cancer "Persister Cells" Tolerant to EGFR Tyrosine Kinase Inhibitors. Cancer Res 2018;78:1044-57.

20. Taniguchi H, Yamada T, Wang R, et al. AXL confers intrinsic resistance to osimertinib and advances the emergence of tolerant cells. Nat Commun 2019;10:259. 
21. Hata AN, Niederst MJ, Archibald HL, et al. Tumor cells can follow distinct evolutionary paths to become resistant to epidermal growth factor receptor inhibition. Nat Med 2016;22:262-9.

22. El Kadi N, Wang L, Davis A, et al. The EGFR T790M Mutation Is Acquired through AICDA-Mediated Deamination of 5-Methylcytosine following TKI Treatment in Lung Cancer. Cancer Res 2018;78:6728-35.

23. Shah KN, Bhatt R, Rotow J, et al. Aurora kinase A drives the evolution of resistance to third-generation EGFR inhibitors in lung cancer. Nat Med 2019;25:111-8.

24. Tanaka K, Han S, YT G, et al. Enhancin apoptosis to improve the therapy of EFR-mutant lung cancer. AACR Annual Meeting 2019.

25. Malumbres M, Perez de Castro I. Aurora kinase A inhibitors: promising agents in antitumoral therapy. Expert
Opin Ther Targets 2014;18:1377-93.

26. Willems E, Dedobbeleer M, Digregorio M, et al. The functional diversity of Aurora kinases: a comprehensive review. Cell Div 2018;13:7.

27. Wu CC, Yu CT, Chang GC, et al. Aurora-A promotes gefitinib resistance via a NF-kappaB signaling pathway in p53 knockdown lung cancer cells. Biochem Biophys Res Commun 2011;405:168-72.

28. Katsha A, Belkhiri A, Goff L, et al. Aurora kinase A in gastrointestinal cancers: time to target. Mol Cancer 2015;14:106.

29. Li Y, Zhou W, Tang K, et al. Silencing Aurora A leads to re-sensitization of breast cancer cells to Taxol through downregulation of SRC-mediated ERK and mTOR pathways. Oncol Rep 2017;38:2011-22.
Cite this article as: Suda K. Targeting the reversible drugtolerant state: Aurora kinase A, is that the final answer? Transl Cancer Res 2019;8(Suppl 6):S564-S568. doi: 10.21037/ tcr.2019.05.21 